\title{
CRESCIMENTO E NUTRIÇÃO MINERAL DE MUDAS DE UMBUZEIRO (Spondias tuberosa Arr. Cam.) SUBMETIDAS A NÍVEIS DE SALINIDADE EM SOLUÇÃO NUTRITIVA ${ }^{1}$
}

\author{
Growth and mineral nutrition of umbuzeiro seedlings \\ (Spondias tuberosa Arr. Cam.) under salt stress in nutrient solution
}

Orlando Sílvio Caires Neves ${ }^{2}$, Janice Guedes de Carvalho ${ }^{3}$, Carlos Ribeiro Rodrigues ${ }^{2}$

\begin{abstract}
RESUMO
Para avaliar o crescimento e a nutrição mineral de mudas de umbuzeiro em solução nutritiva com diferentes níveis de $\mathrm{NaCl}$, conduziu-se este experimento em casa-de-vegetação do Departamento de Ciência do Solo da Universidade Federal de Lavras. Adotou-se o delineamento em blocos casualizados com quatro repetições e seis doses de $\mathrm{NaCl}(0,20,40,60,80$ e 100 $\mathrm{mmol} \mathrm{L}{ }^{-1}$ ). As mudas foram adaptadas na solução de Hoagland a 25\%, 33\% e 50\% da sua força iônica, permanecendo durante 15 dias em cada concentração. Após esse período, conduziram-se as plantas por 60 dias em solução com 100\% da força iônica e os tratamentos com $\mathrm{NaCl}$. A troca das soluções foi feita quinzenalmente. $\mathrm{O} \mathrm{NaCl}$ na solução nutritiva afetou negativamente o crescimento das mudas de umbuzeiro. O umbuzeiro foi classificado como moderadamente tolerante à salinidade quando cultivado em até $31 \mathrm{mmol} \mathrm{L}^{-1}$ de $\mathrm{NaCl}$ na solução nutritiva, sendo a compartimentalização nas raízes o possível mecanismo de tolerância à salinidade. A ordem de acumulação dos macronutrientes foi: $\mathrm{N}>\mathrm{K}>\mathrm{Ca}>\mathrm{P}>\mathrm{S}>\mathrm{Mg}$ e $\mathrm{N}>\mathrm{Ca}>\mathrm{K}>\mathrm{P}>\mathrm{S}>$ $\mathrm{Mg}$, na ausência e com $100 \mathrm{mmol} \mathrm{L}^{-1}$, respectivamente, e a dos micronutrientes, exceto o $\mathrm{Cl}$, foi: $\mathrm{Fe}>\mathrm{B}>\mathrm{Mn}>\mathrm{Zn}>\mathrm{Cu}$, independente da concentração de $\mathrm{NaCl}$ no meio.
\end{abstract}

Termos para indexação: Spondias tuberosa, umbuzeiro, salinidade, translocação, acúmulo, cloreto de sódio.

\begin{abstract}
To evaluate the growth and the mineral nutrition of umbuzeiro seedlings, under salt stress, an experiment in nutrient solution was conducted, under greenhouse conditions in the Soil Science Department of the Federal University of Lavras, Brazil. The experimental design was randomized blocs, with four replications and six doses of $\mathrm{NaCl}\left(0,20,40,60,80\right.$, and $100 \mathrm{mmol} \mathrm{L}^{-}$ ${ }^{1}$ ). The seedlings were adapted in the Hoagland solution to 25,33 and $50 \%$ of ionic forces, staying for fifteen days in each concentration. After this period the seedlings were conduced for 60 days in nutrient solution with $100 \%$ of the ionic force and the treatments with $\mathrm{NaCl}$. The change of the solutions was made biweekly. The $\mathrm{NaCl}$ in the nutrient solution affected negatively the growth of the umbuzeiro seedlings. The umbuzeiro was classified as moderately tolerant to salinity when cultivated below 31 $\mathrm{mmol} \mathrm{L}{ }^{-1}$ of $\mathrm{NaCl}$. The roots compartmentalization is the possible mechanism of tolerance to salt in umbuzeiro seedlings. The order of macronutrient accumulation was: $\mathrm{N}>\mathrm{K}>\mathrm{Ca}>\mathrm{P}>\mathrm{S}>\mathrm{Mg}$ and $\mathrm{N}>\mathrm{Ca}>\mathrm{K}>\mathrm{P}>\mathrm{S}>\mathrm{Mg}$, in the absence and with 100 mmol $\mathrm{L}^{-1}$, respectively and the micronutrient accumulation, except $\mathrm{Cl}$, was: $\mathrm{Fe}>\mathrm{B}>\mathrm{Mn}>\mathrm{Zn}>\mathrm{Cu}$, independent of the concentration of $\mathrm{NaCl}$ in the solution.
\end{abstract}

Index terms: Spondias tuberosa, umbuzeiro, salinity, translocation, accumulation, sodium chlorine.

(Recebido para publicação em 31 de março de 2003 e aprovado em 17 de agosto de 2004)

\section{INTRODUÇÃO}

O umbuzeiro (Spondias tuberosa Arr. Cam.) é uma frutífera adaptada a sobreviver e produzir sob condição de estresse hídrico. Apesar de sua distribuição ser dispersa, consagra-se como uma espécie frutífera de grande importância econômica, social e ecológica para o semi-árido nordestino (SILVA et al., 1987b). A comercialização dos frutos, colhidos de forma extrativista, representa uma fonte de renda importante para muitas famílias nordestinas, chegando a contribuir com até a metade da renda média anual (GONDIM et al., 1991). Assim, pode-se pensar no umbuzeiro como uma alternativa viável para o desenvolvimento agrícola da região semi-árida do Nordeste do Brasil.

Para ressaltar a importância econômica do umbuzeiro, Silva et al. (1987b) destacaram alguns subprodutos obtidos do processamento do fruto ou do xilopódio (órgão de reserva de água e nutrientes), tais como: doces, farinha, gelatina, umbuzada, acetona, torta para

\footnotetext{
. Extraído da dissertação de mestrado apresentada pelo primeiro autor à Universidade Federal de Lavras/UFLA - Caixa Postal 3037 - $37200-000$ - Lavras, MG

2. Alunos do Curso de Doutorado em Solos e Nutrição de Plantas da UFLA.

3. Professora Titular do Departamento de Ciência do Solo da UFLA
} 
animais, suco medicinal da raiz, polpa, vinagre, vinho, etc. $\mathrm{O}$ fruto in natura é muito apreciado pela população nordestina.

A tolerância de uma planta ao estresse salino varia entre as espécies e, também, com relação ao sal predominante. Diversos íons estão envolvidos no processo de salinização dos solos, destacando-se: cloreto, sulfato, carbonato, sódio, cálcio, magnésio e, mais raramente, nitrato e potássio. De acordo com o nível de tolerância à salinidade, as plantas são classificadas em: halófitas ou natrofílicas (apresentam aumento de crescimento em baixas concentrações salinas, com decréscimo de seu desenvolvimento em altas concentrações); tolerantes a sais não-halófitas (mantêm seu crescimento em baixas concentrações de sais, mas diminuem seu crescimento em altas concentrações); sensíveis a sais (têm seu crescimento diminuído em baixas concentrações de sais); e muito sensíveis à salinidade (absorvem água do solo juntamente com os sais, possibilitando a toxidez na planta pelo excesso de sal) (LIMA, 1997).

Segundo observações de Tyerman e Skerrett (1999), em ambientes salinos, o $\mathrm{NaCl}$ tem-se mostrado como sendo o sal predominante, causando a maioria das injúrias nas plantas. $\mathrm{O}$ efeito osmótico tem sido a causa maior da redução do crescimento das plantas, associado à toxidez de íons pela absorção excessiva de sódio e cloro e ao desequilíbrio nutricional causado pelos distúrbios na absorção e/ou distribuição dos nutrientes (YAHYA, 1998). As diferenças são grandes entre as espécies de plantas na habilidade para prevenir ou tolerar elevadas concentrações de $\mathrm{Na}$ nas folhas (MUNNUS, 1993, citado por FERNANDES et al., 2002).

Objetivou-se com este trabalho avaliar o crescimento e nutrição de mudas de umbuzeiro em solução nutritiva com diferentes doses de $\mathrm{NaCl}$.

\section{MATERIAL E MÉTODOS}

O experimento foi conduzido de janeiro a junho de 2002, na casa-de-vegetação do Departamento de Ciência do Solo da Universidade Federal de Lavras (Lavras-MG), definida geograficamente pelas coordenadas de $21^{\circ} 14^{\prime}$ de latitude sul e $45^{\circ} 00^{\prime} \mathrm{de}$ longitude oeste, com altitude de $910 \mathrm{~m}$.

Adotou-se o delineamento em blocos casualizados com quatro repetições e seis doses de $\mathrm{NaCl}$ $\left(0,20,40,60,80\right.$ e $\left.100 \mathrm{mmol} \mathrm{L} \mathrm{L}^{-1}\right)$. As doses corresponderam a valores de condutividade elétrica (CE) de: $2,05,4,06,5,86,7,62,9,42$ e $11,28 \mathrm{dS} \mathrm{m}^{-1}$, respectivamente. As mudas para a implantação do experimento foram oriundas de sementeira (vermiculita), e as sementes do umbuzeiro passaram pelo processo de quebra de dormência mediante escarificação mecânica, conforme recomendação de Nascimento et al. (2000). Após 15 dias da emergência, as mudas foram transferidas para solução de Hoagland (HOAGLAND e ARNON, 1950) a $25 \%, 33 \%$ e $50 \%$ da sua força iônica, permanecendo durante um período de 15 dias em cada concentração. Após o período de adaptação, foram adicionados à solução de trabalho (Hoagland a 100\% da força) os tratamentos com $\mathrm{NaCl}$. Cada parcela foi constituída por uma planta em vaso de 3 litros. A troca das soluções foi feita quinzenalmente durante os 60 dias de condução do experimento. A solução nutritiva foi mantida sob aeração constante durante todo o período experimental, bem como foi mantido o volume dos vasos sempre em 3 litros pela reposição diária com água deionizada. As mudas foram transplantadas para os vasos com os tratamentos, com uma altura média de 41 $\mathrm{cm}$ e diâmetro médio do caule de $3,2 \mathrm{~mm}$.

Foram avaliados os dados referentes ao incremento em altura e diâmetro do caule, que consistiram nas diferenças entre a primeira e a última medida realizada no experimento. Depois de colhido, o material vegetal foi separado em raízes, caule e folhas e seco em estufa a $70^{\circ} \mathrm{C}$, até peso constante. A matéria seca correspondente a cada uma das partes foi pesada e pela soma delas, foi obtida a matéria seca total. A relação raiz/parte aérea também foi calculada.

No extrato nítrico-perclórico, foram determinados os teores de $\mathrm{P}$ por colorimetria; $\mathrm{Ca}, \mathrm{Mg}$, $\mathrm{Cu}, \mathrm{Fe}, \mathrm{Mn}$ e $\mathrm{Zn}$, por espectrofotometria de absorção atômica; $\mathrm{K}$ e $\mathrm{Na}$, por fotometria de chama e $\mathrm{S}$, por turbidimetria. Os teores de $\mathrm{N}$ total foram determinados pelo método semimicro Kjeldahl. O B, após digestão por via seca, foi determinado por colorimetria (método da curcumina) e o $\mathrm{Cl}$ foi determinado por titulação com nitrato de prata (MALAVOLTA et al., 1997).

A acumulação dos nutrientes e do sódio foi determinada pela soma dos acúmulos nas diferentes partes da planta, que, por sua vez, foram calculados com base nos teores e nas produções de matéria seca. A translocação foi calculada pela seguinte fórmula: translocação $(\%)=$ (acúmulo do nutriente na parte aérea/ acúmulo total do nutriente na planta) x 100 .

A tolerância à salinidade foi avaliada com base na redução da produção de matéria seca, segundo critérios estabelecidos por Ponnamperuma (1977), citado por Fageria (1984), em que a planta é tida como sensível, moderadamente sensível, moderadamente tolerante ou tolerante com reduções 
na matéria seca de >60, 41-60, 21-40 e 0-20\%, respectivamente.

Os resultados foram submetidos à análise de variância, e como ocorreram diferenças significativas pelo teste $\mathrm{F}(\mathrm{P}<0,05)$, foram ajustados modelos de regressão utilizando-se o programa estatístico SISVAR (FERREIRA, 2000).

\section{RESULTADOS E DISCUSSÃO}

As concentrações de $\mathrm{NaCl}$ na solução nutritiva influenciaram significativamente as variáveis avaliadas no experimento. $\mathrm{O}$ aumento das doses de $\mathrm{NaCl}$ na solução promoveu uma redução no crescimento da plantas em relação ao controle. $\mathrm{Na}$ ausência de $\mathrm{NaCl}$ na solução, durante o período experimental, o incremento em altura foi de $21,54 \mathrm{~cm}$, sendo esse valor reduzido em $50 \%$ na dose de $27,3 \mathrm{mmol} \mathrm{L}^{-1}$ de $\mathrm{NaCl}$, segundo um modelo quadrático (Figura 1 a).

O diâmetro do caule (Figura $1 \mathrm{~b}$ ) apresentou uma redução de $50 \%$ na dose de $56,5 \mathrm{mmol} \mathrm{L}^{-1}$ de $\mathrm{NaCl}$, em comparação à testemunha, decrescendo linearmente
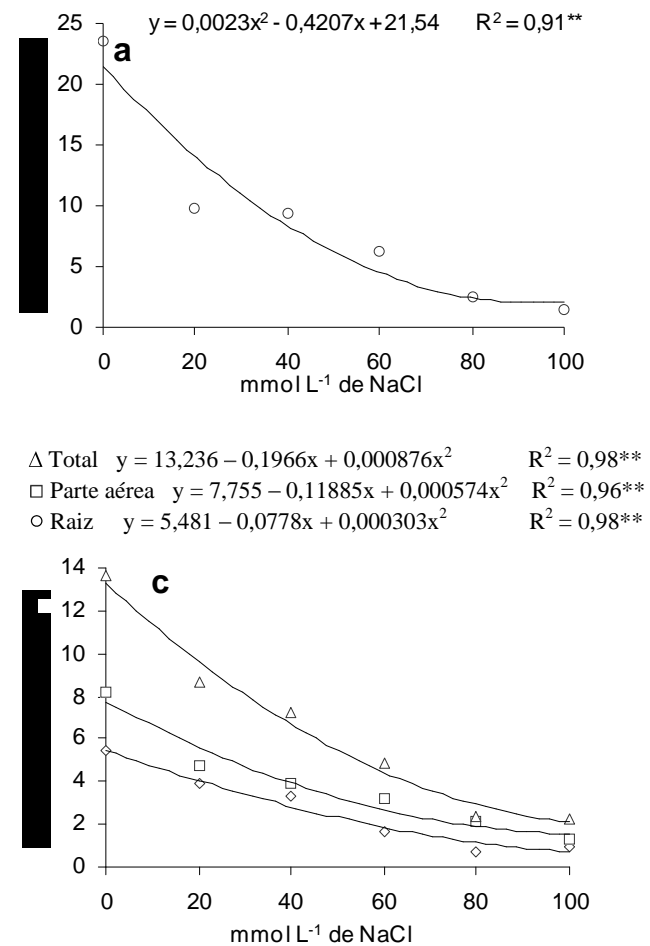

com o aumento da salinidade. $\mathrm{O}$ crescimento das plantas em altura foi mais prejudicado com o aumento inicial da salinidade do que o diâmetro do caule, embora ambos tenham sofrido reduções (Figuras $1 \mathrm{a}, \mathrm{b}$ ).

Dados obtidos por Lima (1999) demonstram o efeito negativo de sais sobre o crescimento inicial da pinheira (Annona squamosa L.), sendo os sais $\mathrm{Na}_{2} \mathrm{SO}_{4} \mathrm{e}$ $\mathrm{NaCl}$ aqueles que mais limitaram o crescimento na fase de muda.

Apesar da grande redução no crescimento, apenas a partir da dose de $60 \mathrm{mmol} \mathrm{L}^{-1}$ de $\mathrm{NaCl}$ é que as plantas apresentaram sintomas visuais de toxidez, os quais foram caracterizados por clorose, seguida de necrose no ápice e margens das folhas mais velhas, desfolha e morte de plantas na dose máxima testada (100 mmol $\mathrm{L}^{-1}$ de $\mathrm{NaCl}$ ). Silva et al. (1987a) trataram pinheiras com $0,20,40,60,80$ e $100 \mathrm{mg} \mathrm{m}^{-2}$ de $\mathrm{NaCl}$ incorporados ao solo e, após quatro meses, todas as plantas, exceto aquelas do tratamento testemunha, sofreram abscisão precoce das folhas mais velhas.

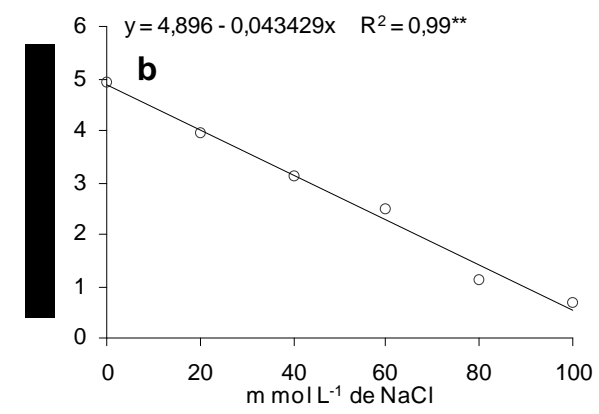

$y=0,7924-0,0026 x \quad R^{2}=0,51^{* *}$

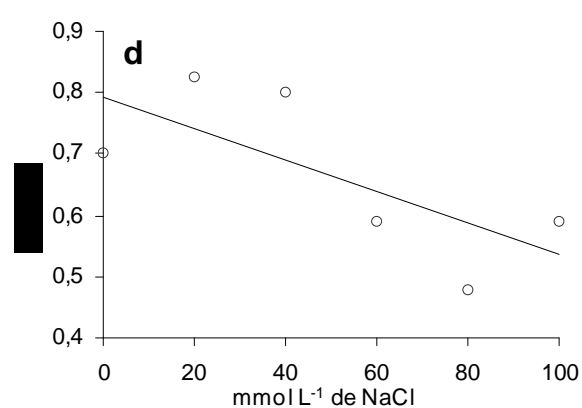

FIGURA 1 - Incremento em altura (a), incremento no diâmetro do caule (b), matéria seca total - MST, da parte aérea - MSPA e das raízes - MSR (c) e relação raiz/parte aérea (R/PA) (d) de mudas de umbuzeiro em função de doses de $\mathrm{NaCl}$ na solução nutritiva. (**) significativo a $1 \%$. 
A redução ou inibição do crescimento das plantas pela salinidade tem sido atribuída ao efeito osmótico, associado à toxidez pela absorção excessiva de $\mathrm{Na}$ e $\mathrm{Cl}$ e ao desequilíbrio nutricional causado pelos distúrbios na absorção e distribuição dos nutrientes (YAHYA, 1998).

As produções de matéria seca da parte aérea, de raiz e total (Figura 1 c) seguiram modelos de resposta quadráticos, com redução nos valores dessas variáveis à medida que se aumentaram as doses de $\mathrm{NaCl}$ na solução. Esse comportamento concorda com o descrito por Cordeiro (1997) para mudas de mamoeiro Havaí; por Fernandes et al. (2002) para pupunheira submetida a níveis de salinidade em solução nutritiva e por Miranda et al. (2002), para cajueiro. Comparativamente à testemunha, a matéria seca total foi reduzida em $82,3 \%$ na dose de $100 \mathrm{mmol} \mathrm{L}^{-1}$ de $\mathrm{NaCl}$ e atingiu $50 \%$ de produção a uma dose de 41,2 $\mathrm{mmol} \mathrm{L}^{-1}$ de $\mathrm{NaCl}$. Nessa dose, a condutividade elétrica (CE) foi equivalente a $6,04 \mathrm{dS} \mathrm{m}^{-1}$.

Utilizando os critérios descritos por Ponnamperuma (1977), citado por Fageria (1984), para classificação de plantas quanto à sua tolerância à salinidade, como verificado na Tabela 1 , pode-se considerar, com base na produção de matéria seca total obtida, que a muda de umbuzeiro é tolerante se cultivada em ambientes onde há presença de $\mathrm{NaCl}$ até o nível $16 \mathrm{mmol} \mathrm{L}^{-1}$, pois, nesse nível de salinidade, a redução na produção de matéria seca foi de até $20 \%$; moderadamente tolerante entre 16,1 e $31 \mathrm{mmol} \mathrm{L}^{-1}$ de $\mathrm{NaCl}$, sendo a redução nesse nível de $20 \%$ a $40 \%$; moderadamente sensível entre 31,1 e $52,9 \mathrm{mmol} \mathrm{L}^{-1}$ de $\mathrm{NaCl}$, com redução de $41 \%$ a $60 \%$ e sensível em níveis iguais ou superiores a $53 \mathrm{mmol} \mathrm{L}^{-1}$ de $\mathrm{NaCl}$, com reduções acima a $60 \%$. Isso corresponde, em termos de $\mathrm{CE}$, a 2,$34 ; 4,54 ; 7,76$ e 7,76 $\mathrm{dS} \mathrm{m}^{-1}$, respectivamente, para cada classe de tolerância. Adicionando-se a essa classificação a de Mass (1986), em que, segundo seus critérios, uma planta é tida como moderadamente tolerante à salinidade quando cultivada em ambientes com CE entre 3 e $6 \mathrm{dS} \mathrm{m}^{-1}$, com reduções na matéria seca inferiores a $40 \%$, a muda de umbuzeiro pode ser considerada como moderadamente tolerante à salinidade. Richards (1954) estabelece critério para a salinidade dos solos onde o mesmo é tido como salino em CE a partir de $4 \mathrm{dS} \mathrm{m}^{-1}$, assim, pode-se inferir que em um solo salino com CE por volta de $4 \mathrm{dS} \mathrm{m}^{-1}$, as mudas de umbuzeiro aprestariam uma tolerância moderada.

Campos (2001) relata o fato de que as plantas podem, até certo ponto, desenvolver adaptações para resistirem melhor a solos salinos, apresentando um incremento na pressão osmótica da parte aérea e das raízes que contrabalance o aumento da pressão osmótica da solução do solo.

Segundo Muns (1993), a parte aérea das plantas é mais sensível ao estresse salino do que as raízes devido ao desequilíbrio entre os cátions, em conseqüência de complexas interações no sistema de transporte. No caso do umbuzeiro, em razão de todo o aparato desenvolvido no seu sistema radicular para adaptação à seca, esperava-se que as raízes fossem menos prejudicadas pela presença do sal $(\mathrm{NaCl})$ do que a parte aérea, o que não aconteceu. Tanto a parte aérea, quanto as raízes foram afetadas negativamente pelo estresse salino, sendo o estresse sofrido pelas raízes maior (Figura 1 c). Fernandes et al. (2002), trabalhando com a pupunheira e Araújo Filho et al. (1995), com bananeira, também constataram haver influência negativa do estresse salino tanto para a parte aérea quanto para as raízes dessas plantas.

Quando uma planta é submetida a determinados tipos de estresse, ela aloca reservas para os órgãos que estão diretamente ligados a esse estresse, como uma estratégia de sobrevivência (FERRI, 1979). Exemplo disso é quando a planta, para se adaptar ao estresse hídrico, favorece o desenvolvimento do sistema radicular, ou seja, aumenta a relação raiz/parte aérea.

TABELA 1 - Classificação das mudas de umbuzeiro quanto à tolerância à salinidade em solução nutritiva.

\begin{tabular}{|c|c|c|c|}
\hline Classificação & $\begin{array}{c}\text { Redução na produção da } \\
\text { matéria seca }(\%)\end{array}$ & $\mathrm{NaCl}\left(\mathrm{mmol} \mathrm{L}^{-1}\right)$ & $\begin{array}{l}\text { Produção de matéria seca } \\
\left.\text { apresentada (g planta }^{-1}\right)\end{array}$ \\
\hline Tolerante & $\leq 20$ & $\leq 16,0$ & $13,24-10,60$ \\
\hline Moderadamente tolerante & -40 & $16,1-31,0$ & $10,59-7,98$ \\
\hline Moderadamente sensível & -59 & $31,1-52,9$ & $7,97-5,28$ \\
\hline Sensível & $\geq 60$ & $\geq 53,0$ & $<5,28$ \\
\hline
\end{tabular}


No caso do estresse salino, o umbuzeiro apresentou comportamento inverso, ou seja, com o aumento dos níveis de salinidade, a raiz foi mais prejudicada do que a parte aérea, razão pela qual houve redução na relação $\mathrm{R} / \mathrm{PA}$ de 0,79 na testemunha para 0,53 na dose de $100 \mathrm{mmol} \mathrm{L}^{-1}$ de $\mathrm{NaCl}$ (Figura $1 \mathrm{~d}$ ), mostrando, assim, não ter havido maior alocação de compostos nas raízes. O menor crescimento das raízes pode ter sido uma adaptação das mudas de umbuzeiro, as quais, com uma redução na superfície de absorção, estariam excluindo parte do $\mathrm{Na}$ e do $\mathrm{Cl}$ da sua absorção.

À exceção do cloro, o aumento das doses de $\mathrm{NaCl}$ na solução nutritiva promoveu redução no acúmulo dos nutrientes nas plantas de umbuzeiro (Figura 2). Esse fato é explicado pela redução na produção de matéria seca das plantas no mesmo sentido (Figura $1 \mathrm{c}$ ); o fato de o cloro ter sua concentração aumentada na solução nutritiva com o aumento das doses de $\mathrm{NaCl}$ pode explicar esse comportamento. Comparando-se os tratamentos testemunha e a maior dose de $\mathrm{NaCl}$ utilizada (100 mmol $\mathrm{L}^{-1}$ ), a redução de acumulação dos nutrientes foi de: $\mathrm{N}=77, \mathrm{P}=81, \mathrm{~K}=84, \mathrm{Ca}$ e $\mathrm{Mg}=78, \mathrm{~S}=83$, $\mathrm{B}=82, \mathrm{Cu}=69, \mathrm{Mn}=52, \mathrm{Zn}=44$ e $\mathrm{Fe}=35 \%$. Nessa relação, observou-se que o K para os macro e o $\mathrm{B}$ para os micronutrientes foram os mais afetados pelo estresse salino. Fernandes et al. (2002) estudaram o acúmulo de macronutrientes e $\mathrm{Na}$ na pupunheira e também constataram uma redução na absorção de $\mathrm{K}$ quando se adicionou $\mathrm{Na}$ na solução nutritiva e atribuíram esse efeito ao antagonismo existente entre esses cátions. Miranda et al. (2002) observaram que, segundo alguns autores, o $\mathrm{NaCl}$, além de inibir a translocação do $\mathrm{Ca}$ na planta, pode reduzir sua disponibilidade na solução, devido à precipitação com o $\mathrm{Cl}$.

As doses de $\mathrm{NaCl}$ em solução que promoveram uma redução de $50 \%$ na acumulação foram de: 40,0 ; 60,$6 ; 48,5 ; 46,8 ; 42,2$ e $59,1 \mathrm{mmol} \mathrm{L}^{-1}$ de $\mathrm{NaCl}$, respectivamente, para $\mathrm{N}, \mathrm{P}, \mathrm{K}, \mathrm{Ca}, \mathrm{Mg}$ e $\mathrm{S} . \mathrm{A}$ redução do acúmulo dos nutrientes com o aumento das doses de $\mathrm{NaCl}$ na solução foi influenciada pela diminuição na produção de matéria seca, que apresentou comportamento semelhante. Segundo Faquin (2001), o acúmulo de macronutriente segue a mesma tendência da produção de matéria seca. Esse autor cita que para plantas de soja cultivadas em ambientes não-salinos, há uma perfeita sobreposição das curvas de produção de matéria seca e acumulação de macronutrientes.
Para os macronutrientes, na ausência de $\mathrm{NaCl}$, a ordem decrescente de acumulação foi, em mg planta ${ }^{-1}$ : $\mathrm{N}(279,7)>\mathrm{K}(271,4)>\mathrm{Ca}(206,7)>\mathrm{P}(132,5)>\mathrm{S}$ $(26,1)>\mathrm{Mg}(18,9)$. Na dose máxima de $\mathrm{NaCl}$, essa ordem foi alterada para $\left(\mathrm{mg} \mathrm{planta}^{-1}\right): \mathrm{N}(76,8)>\mathrm{Ca}$ $(47,5)>\mathrm{K}(43,5)>\mathrm{P}(25,1)>\mathrm{S}(4,4)>\mathrm{Mg}(4,1)$. Isso provavelmente se deveu à substituição do $\mathrm{K}$ pelo $\mathrm{Na}$, fazendo com que a absorção do nutriente fosse diminuída quando a planta foi submetida às condições de estresse salino e também uma tentativa de alocar mais Ca para minimizar problemas de membranas, principalmente nas folhas. Em algumas plantas, a substituição de parte do $\mathrm{K}$ por Na pode estimular o crescimento (MALAVOLTA et al., 1997), o que, para o umbuzeiro, não foi verificado.

De forma decrescente, a acumulação dos micronutrientes (exceto o $\mathrm{Cl}$ ) pelas mudas na ausência de $\mathrm{NaCl}$ foi $\left(\mathrm{mg} \mathrm{planta}^{-1}\right)$ : $\mathrm{Fe}(8,92)>\mathrm{B}(0,43)>\mathrm{Mn}$ $(0,15)>\mathrm{Zn}(0,13)>\mathrm{Cu}(0,07)$. Essa ordem foi mantida também na dose máxima de $\mathrm{NaCl}$ estudada, excetuando a acumulação de $\mathrm{Mn}$ e $\mathrm{Zn}$, que nesse nível foram iguais $\left(0,07 \mathrm{mg}\right.$ planta $\left.^{-1}\right)$. $\mathrm{O}$ acúmulo do $\mathrm{Fe}$, além de ser o mais elevado entre os micronutrientes (exceto o $\mathrm{Cl}$ ), foi o menos afetado pela salinidade. As doses de $\mathrm{NaCl}$ em solução que provocaram redução de $50 \%$ no acúmulo dos micronutrientes (exceto $\mathrm{Cl}$ ) foram de 60,3 (B); $70,4(\mathrm{Cu})>100$ (Fe e $\mathrm{Zn})$ e $89,6 \mathrm{mmol} \mathrm{L}^{-1}(\mathrm{Mn})$. Comparativamente com os macronutrientes, o acúmulo dos micronutrientes foi menos prejudicado pela salinidade.

Os acúmulos de $\mathrm{Cl}$ e $\mathrm{Na}$ ajustaram-se a funções quadráticas com aumento da absorção nas primeiras doses de $\mathrm{NaCl}$. Para o $\mathrm{Cl}$, o aumento se deu até a dose de $69,7 \mathrm{mmol} \mathrm{L}^{-1}$ de $\mathrm{NaCl}$, e para o $\mathrm{Na}$, até a dose de $61,7 \mathrm{mmol} \mathrm{L}^{-1}$ de $\mathrm{NaCl}$, passando a decair a partir dessas doses. Essa queda se deveu principalmente à diminuição da produção de matéria seca, pois os teores, tanto de $\mathrm{Cl}$ quanto de $\mathrm{Na}$, aumentaram com o maior nível de salinidade. $\mathrm{O}$ acúmulo de $\mathrm{Cl}$ foi maior que a de $\mathrm{Na}$ em todos os níveis de salinidade (Figura $2 \mathrm{f}$ ). Fernandes et al. (2002) também verificaram maior acúmulo de $\mathrm{Cl}$ do que $\mathrm{Na}$ em pupunheira, em condições salinas.

$\mathrm{Na}$ dose de $\mathrm{NaCl}$ que promoveu maior acúmulo de $\mathrm{Na}$, o conteúdo de $\mathrm{Cl}$ e $\mathrm{Na}$ foram respectivamente de 153,56 e 68,67 g planta $^{-1}$, equivalentes em $\mathrm{mM}$ a 4,33 e 2,99 . A sintomatologia apresentada nas folhas mais velhas do umbuzeiro é característica da toxidez de $\mathrm{Na}$, significando que a maior parte dos prejuízos do umbuzeiro devem ter sido provocados por esse elemento. Segundo Malavolta et al. (1997), em plantas sensíveis de soja 
(Paraná), o teor foliar de $\mathrm{Cl}$ é superior a $30 \mathrm{~g} \mathrm{~kg}^{-1} \mathrm{e}$, em plantas tolerantes (IAC-3), é de até $2 \mathrm{~g} \mathrm{~kg}^{-1}$, o que caracteriza a exclusão de $\mathrm{Cl}$ das folhas. Neste estudo, a maior concentração de $\mathrm{Cl}$ foi atingida na máxima dose de $\mathrm{NaCl}$ testada e nas folhas $\left(84,2 \mathrm{~g} \mathrm{~kg}^{-1}\right)$, dose essa que proporcionou a morte das plantas. $\mathrm{Na}$ dose de $60 \mathrm{mmol}$
$\mathrm{L}^{-1}$ de $\mathrm{NaCl}$, em que aparecerem os primeiros sintomas foliares de toxidez, o acúmulo de $\mathrm{Cl}$ nas folhas foi de $53,3 \mathrm{~g} \mathrm{~kg}^{-1}$. Segundo Alam (1994), o maior acúmulo de $\mathrm{Cl}$ pode indicar uma maior sensibilidade das plantas a danos por $\mathrm{Cl}$ do que por $\mathrm{Na}$.
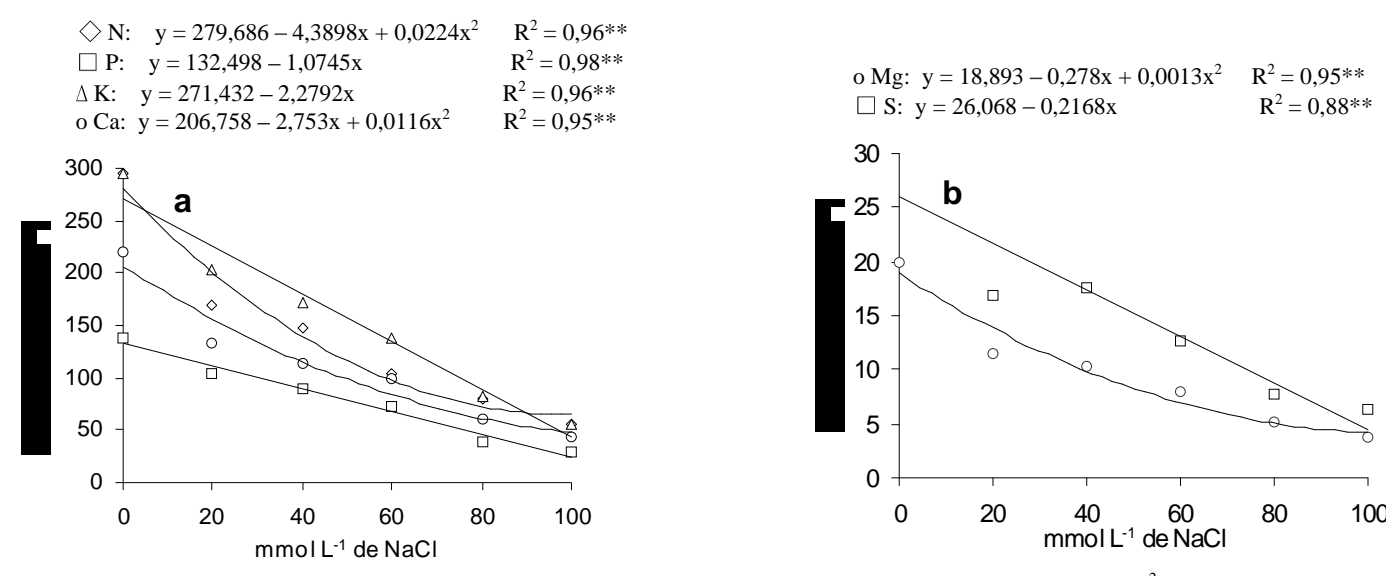

o B: $y=0,433-0,00353 x \quad R^{2}=0,85$
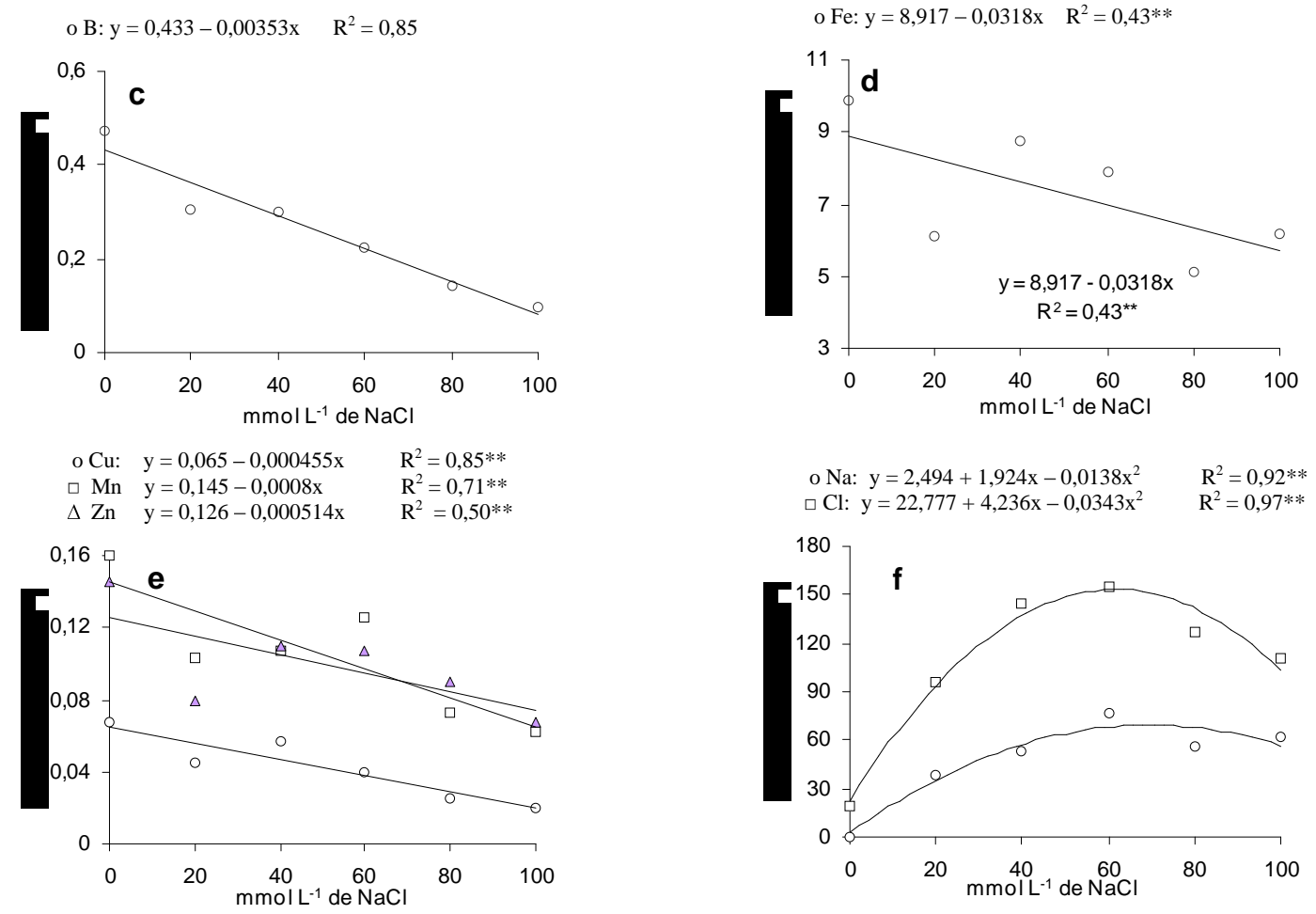

FIGURA 2 - Acumulação de N, P, K e Ca (a); Mg e S (b); B (c); Fe (d); Cu, Mn e Zn (e) e; Na e Cl (f) por mudas de umbuzeiro em função de doses de $\mathrm{NaCl}$ na solução nutritiva. (**) significativo a $1 \%$. 
A translocação, ou seja, o percentual do elemento absorvido que segue para a parte aérea é uma boa medida para caracterizar a acumulação do nutriente nas raízes ou na parte aérea, auxiliando, assim, na identificação de possíveis mecanismos envolvidos na tolerância de plantas submetidas a ambientes salinos, tais como a compartimentalização e a interação com outros elementos.

Floweres et al. (1977) observaram que o crescimento de halófitas é, invariavelmente, acompanhado pelo acúmulo de íons nas folhas, enquanto, nas glicófitas, é acompanhado pela exclusão desses, acumulando-os nas raízes e, às vezes, no caule.

As translocações de $\mathrm{N}, \mathrm{P}$ e $\mathrm{K}$ estão representadas na Figura 3 a, e para o N, esses valores não diferiram significativamente entre as doses de $\mathrm{NaCl}$ estudadas. O P translocado passou de $44,8 \%$ na ausência de $\mathrm{NaCl}$ para $54 \%$ na dose mais alta de $\mathrm{NaCl}$; entretanto, devido ao baixo coeficiente de determinação $(0,24)$, fica difícil fazer alguma inferência a respeito desse comportamento.

A translocação de $\mathrm{K}$ ajustou-se a uma equação quadrática, com o máximo translocado $(60,7 \%)$ para a parte aérea, ocorrendo na dose de $90 \mathrm{mmol} \mathrm{L}^{-1}$ de $\mathrm{NaCl}$. A maior parte do $\mathrm{K}$ translocado foi destinada às folhas $(34,5,2,5$ e $63 \%$ do $\mathrm{K}$ acumulado, respectivamente, nas raízes, no caule e nas folhas dados não representados), principalmente nas doses mais baixas, em que o conteúdo de $\mathrm{Na}$ foi relativamente baixo quando comparado a doses maiores. Com esse comportamento infere-se que a planta "tentou" estabelecer um melhor ajuste osmótico no sentido de reduzir os danos às folhas, sendo a compartimentalização do $\mathrm{Na}$ nas raízes (doses baixas) e a maior translocação de $\mathrm{K}$ os possíveis mecanismos.

Na Figura 3 b, observa-se que a translocação para o $\mathrm{Ca}$ foi não-significativa. Esse é um ponto importante que deve ser ressaltado, pois altas concentrações de Na no meio celular promovem danos à membrana (MARSCHNER, 1995) da qual o $\mathrm{Ca}$ faz parte. Para o S, a translocação ajustou-se a um modelo quadrático inverso, ou seja, diminuindo até a dose de 20,6 mmol L $\mathrm{m}^{-1} \mathrm{de} \mathrm{NaCl}$, voltando a aumentar a partir dessa dose. $\mathrm{O} \mathrm{Mg}$ foi translocado em um maior percentual com o aumento das doses de $\mathrm{NaCl}$, passando de $51,1 \%$ no tratamento testemunha para $80,3 \%$ na maior testada.

As translocações de $\mathrm{B}, \mathrm{Cu}$ e Fe (Figura 3 c) apresentam comportamentos distintos. Com o aumento dos níveis salinos, a translocação de $B$ tendeu a um aumento e a de $\mathrm{Cu}$, a uma redução; para o $\mathrm{Fe}$, não se encontrou um ajuste adequado para a relação.

Os dados de translocação de $\mathrm{Cl}$ e $\mathrm{Na}$ (Figura 3 e) demonstram que o $\mathrm{Cl}$ apresenta uma maior mobilidade nas mudas de umbuzeiro que o $\mathrm{Na}$, principalmente nas doses mais baixas de $\mathrm{NaCl}$, e, na dose de $20 \mathrm{mmol} \mathrm{L}^{-1}$ de $\mathrm{NaCl}$, apenas $22 \%$ do $\mathrm{Na}$ encontravam-se na parte aérea. Em contrapartida, o $\mathrm{Cl}$ encontrava-se em 58\%. Com o aumento dos níveis de salinidade, o $\mathrm{Na}$ translocou em maior quantidade ( $63 \%$ na dose de $100 \mathrm{mmol} \mathrm{L}^{-1} \mathrm{de} \mathrm{NaCl}$ ). Já o $\mathrm{Cl}$ translocado passou de $58 \%$ para $75 \%$ nas doses de 20 e 100 mmol $\mathrm{L}^{-1}$ de $\mathrm{NaCl}$, respectivamente. Marschner (1995) relata a tolerância à salinidade que, em espécies cítricas, está mais associada com a exclusão de $\mathrm{Na}^{+} \mathrm{e}^{-}$dos ramos e do tecido foliar do que com a compartimentalização nessas partes e isso é conseguido com a restrição da absorção de sais ou devido à restrição ao seu transporte das raízes para a parte aérea. Como visto na Figura $2 \mathrm{f}$, as mudas não conseguiram excluir o $\mathrm{Na}$ da absorção; entretanto, sua translocação (Figura 3 e) foi baixa até o nível de $40 \mathrm{mmol} \mathrm{L}^{-1}$ de $\mathrm{NaCl}$, o que demonstra que apesar de não conseguir excluí-lo da absorção, a planta utilizou o mecanismo de compartimentalização nas raízes para, assim, diminuir os danos às folhas.

Pode-se perceber que o umbuzeiro, quando foi submetido a baixas concentrações de $\mathrm{NaCl}$, concentrou o $\mathrm{Na}$ absorvido primeiramente nas raízes, translocando pequena quantidade para a parte aérea e, com o aumento da salinidade, a planta não conseguiu excluí-lo da parte aérea. 

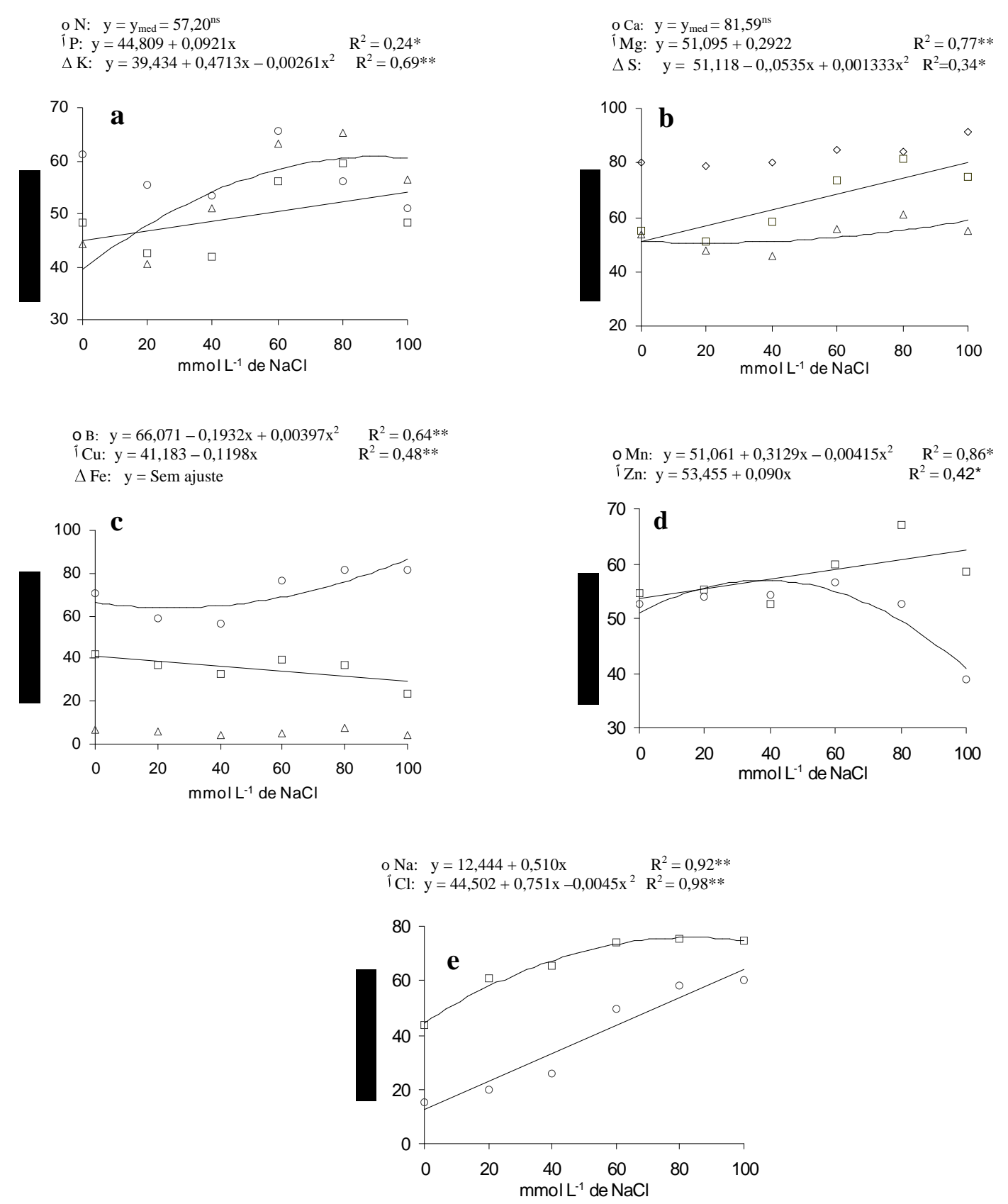

FIGURA 3 - Translocação de N, P, K (a), Ca, Mg, S (b), B, Cu, Fe (c), Mn, Zn (d) e Cl, Na (e) em mudas de umbuzeiro em função de doses de $\mathrm{NaCl}$ na solução nutritiva. ${ }^{\text {ns. } ; ~ * ~ e ~ * *, ~ n a ̃ o-s i g n i f i c a t i v o ~ e ~ s i g n i f i c a t i v o ~ a ~} 5$ e $1 \%$, respectivamente. 


\section{CONCLUSÕES}

a) $\mathrm{O} \mathrm{NaCl}$ na solução nutritiva afetou negativamente o crescimento das mudas de umbuzeiro.

b) $\mathrm{O}$ umbuzeiro foi classificado como moderadamente tolerante à salinidade quando cultivado em até $31 \mathrm{mmol} \mathrm{L}^{-1}$ de $\mathrm{NaCl}$ na solução nutritiva, sendo a compartimentalização nas raízes o possível mecanismo dessa tolerância.

c) A ordem de acumulação dos macronutrientes foi: $\mathrm{N}>\mathrm{K}>\mathrm{Ca}>\mathrm{P}>\mathrm{S}>\mathrm{Mg}$ e $\mathrm{N}>\mathrm{Ca}>\mathrm{K}>\mathrm{P}>$

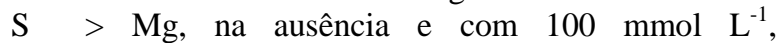
respectivamente.

d) A ordem de acúmulo dos micronutrientes, exceto o $\mathrm{Cl}$, foi: $\mathrm{Fe}>\mathrm{B}>\mathrm{Mn}>\mathrm{Zn}>\mathrm{Cu}$, independente da concentração de $\mathrm{NaCl}$ no meio.

e) A acumulação de $\mathrm{Cl}$ pelo umbuzeiro foi superior à de $\mathrm{Na}$ em todos as doses de $\mathrm{NaCl}$.

f) Houve aumento da translocação de $\mathrm{P}, \mathrm{K}, \mathrm{Mg}$, $\mathrm{S}, \mathrm{B}, \mathrm{Zn}, \mathrm{Cl}$ e $\mathrm{Na}$, diminuição de $\mathrm{Cu}$ e $\mathrm{Mn}$ e alteração pouco significativa de $\mathrm{N}, \mathrm{Ca}$ e $\mathrm{Fe}$, com o aumento das concentrações de $\mathrm{NaCl}$ na solução.

\section{REFERÊNCIAS BIBLIOGRÁFICAS}

ALAM, S. M. Nutrient uptake by plants stress conditions. In: PESSARAKLI, M. Handbook of plant and crop stress. New York: Marcel Dekker, 1994. p. 227-246.

ARAÚJO FILHO, J. B.; GHEYI, H. R.; AZEVEDO, N. C. Tolerância da bananeira à salinidade em fase inicial de desenvolvimento. Pesquisa Agropecuária Brasileira, Brasília, v. 30, n. 7, p. 989-997, jul. 1995.

CAMPOS, C. A. B. Germinação, desenvolvimento e produção do tomateiro industrial, sob estresse salino. 2001. Dissertação (Mestrado) - Universidade Federal de Paraíba, Campina Grande, 2001.

CORDEIRO, J. C. Salinidade da água, fontes e níveis sobre a germinação e formação de mudas de mamoeiro havaí. 1997. Dissertação (Mestrado) Universidade Federal da Paraíba, Areia, 1997.

FAGERIA, N. K. Adubação e nutrição mineral da cultura do arroz. Rio de Janeiro: EMBRAPA, 1984. cap. 10 , p. 302-320.

FAQUIN, V. Nutrição mineral de plantas: textos acadêmicos. Lavras: UFLA/FAEPE, 2001. 182 p.

FERNANDES, A. R.; CARVALHO, J. G.; CURI, N.; PINTO, J. E. B. P.; GUIMARÃES, P. T. G. Nutrição mineral de mudas de pupunheira sob diferentes níveis de salinidade. Pesquisa Agropecuária Brasileira, Brasília, v. 37, n. 11, p. 1613-1619, nov. 2002.

FERREIRA, D. F. Análises estatísticas por meio do SISVAR (Sistema para Análise de Variância) para Windows 4.0. In: REUNIÃO ANUAL DA REGIÃO BRASILEIRA DA SOCIEDADE INTERNACIONAL DE BIOMETRIA, 45., 2000, São Carlos. Anais... São Carlos: UFSCar, 2000. p. 255-258.

FERRI, M. G. Fisiologia vegetal. São Paulo: EPU/USP, 1979. v. 1, 350 p.

FLOWERES, T. J.; TROBE, P. F.; YEO, A. R. The mechanism of salt tolerance in halophytes. Annual Review Phisiology, Palo Alto, v. 28, p. 89-121, 1977.

GONDIM, T. M. S.; SILVA, H.; SILVA, A. Q.; CARDOSO, E. A. Período de ocorrência de formação se xilopódios em plantas de umbu (Spondias tuberosa Arr. Câm. ) propagadas sexualmente e assexuadamente.

A. Revista Brasileira de Fruticultura, Cruz das Almas, v. 13, n. 2, p. 33-38, out. 1991.

HOAGLAND, D. R.; ARNON, D. I. The water culture method of growing plants without soil. Berkeley: University Of California, 1950. 32 p.

LIMA, L. A. Efeitos de sais no solo e na planta. In: CONGRESSO BRASILEIRO DE ENGENHARIA AGRÍCOLA, 26., 1997, Campina Grande. Manejo e controle da salinidade na agricultura irrigada. Campina Grande: UFPB/SBEA, 1997. p. 111-136.

LIMA, K. L. Influência da salinidade da água de irrigação sobre a germinação de sementes $e$ crescimento inicial da pinheira (Annona squamosa L.). 1999. Dissertação (Mestrado) - Universidade Federal da Paraíba, Areia, 1999.

MALAVOLTA, E.; VITTI, G. C.; OLIVEIRA, S. A. Avaliação do estado nutricional das plantas: princípios e aplicações. 2. ed. Piracicaba: POTAFOS, 1997. 319 p.

MARSCHNER, H. Mineral nutrition of higher plants. 2. ed. Academic, 1995. 889 p.

MASS, E. V. Salt tolerance to saline of crops. Applied Agricultural Research, New York, v. 1, n. 1, p. 12-26, 1986.

MIRANDA, J. R. P.; CARVALHO, J. G.; SANTOS, D. R.; FREIRE, A. L. O.; BERTONI, J. C.; MELO, J. R. M.; CALDAS, A. L. Silício e cloreto de sódio na nutrição mineral e produção de matéria seca de plantas de Moringa (Moringa oleifera Lam.). Revista 
Brasileira de Ciência do Solo, Campinas, v. 26, n. 4, p. 957-965, out./dez. 2002.

MUNS, R. Physiological process limiting plant growth in saline soils: some dogmas and hypotheses. Plant, Cell and Environment, Oxford, v. 16, n. 1, p. 15-24, Jan. 1993.

NASCIMENTO, C. E. S.; SANTOS, C. A. F.; OLIVEIRA, V. R. Produção de mudas enxertadas de umbuzeiro (Spondias tuberosa Arruda). Brasília: EMBRAPA Semi-Árido, 2000. 13 p. (Circular técnica, 48).

RICHARDS, L. A. Diagnosis and improvement of saline and alkali soil. Washington: USDA, 1954. 160 p.

SILVA, A. Q.; SILVA, H.; OLIVEIRA, E. M. Sintomas de salinidade em pinha (Annona squamosa L.). In CONGRESSO BRASILEIRO DE INICIAÇÃO CIENTÍFICA EM CIÊNCIAS AGRÁRIAS, 1987, Areia, PB. Anais... Areia: UFPB, 1987a. p. 76.
SILVA, H.; SILVA, A. Q.; OLIVEIRA, A. R.; CAVALCANTE, F. B. Algumas informações pomológicas do umbuzeiro da Paraíba: II. características tecnológicas. In: CONGRESSO BRASILEIRO DE FRUTICULTURA, 9., 1987, Campinas, SP. Resumos... Campinas: SBF, 1987b. v. 1, p. 691-696.

TYERMAN, S. D.; SKERRETT, I. M. Root ion channels and salinity. Scientia Horticulturae, Amsterdam, v. 78, n. 1/4, p. 175-235, Jan. 1999.

YAHYA, A. Salinity effects on growth and on uptake and distribution of sodium and some essential mineral nutrients in sesame. Journal of Plant Nutrition, New York, v. 21, n. 7, p. 1439-1451, 1998. 